\title{
Revolutionising the
} next generation smile - Dr Sandra Tai

\author{
Dr Tai has treated over 2,000 cases with the Invisalign system. She believes that it is the most advanced clear \\ aligner appliance for treating complex cases. In her view, other systems are where Invisalign clear aligners were 15 \\ years ago, only allowing doctors to perform simple tooth movements.
}

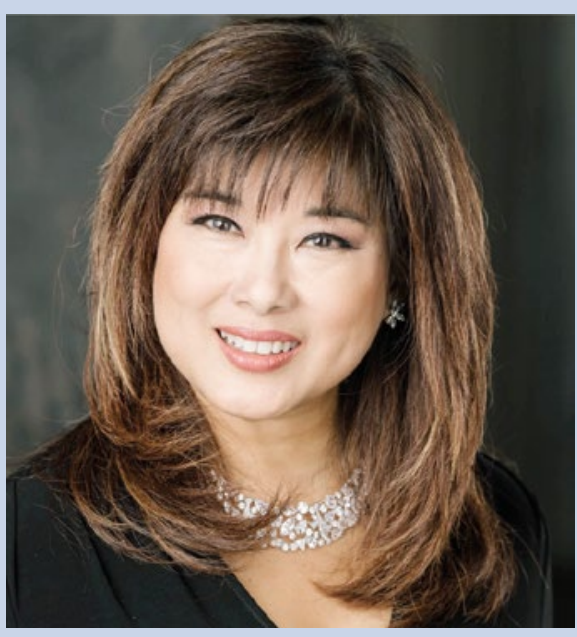

Dr Sandra Tai

'Seventy percent of my patients are growing patients, younger than 20 -years-old. It is actually easier to treat growing patients: you tell them what to do. With adults, it is harder to get them to cooperate. Younger patients are the Generation $\mathrm{Z}$ whose lives revolve around a schedule of school and their hobbies, and the clear aligners are rather easy to fit around their schedules.

'I also find that compliance from young people is very good - it is a generation that is very driven and very focused, they often work towards a goal and clear aligners are what they want. A teenage girl I treated was in the film industry and was about to attend more auditions, so the clear aligners were the solution which allowed her to work and have treatment at the same time. A 9-year-old boy was in martial arts and again the Invisalign clear aligners were the appliance of choice to avoid the risk of injury from facial blows which would be the case with traditional fixed braces. I was initially so surprised to see how younger patients really 'get it' whilst adults or parents are the ones you really need to convince. But actually it makes sense: parents grew up with brackets and wires so it is a different mind-set to a younger generation who have been immersed in digital and almost don't know anything else.

'Patients can be of any age, young or adult. What it is important to realise is that even at a young age, boys and girls are extremely conscious of their appearance and what their teeth look like. Whether they are nine or 50 , patients can show the same personality transformation. They come alive and their self-esteem goes up massively.

'Dentists need to make a proper diagnosis of the problem the patient initially presents with and determine whether it is related to a skeletal (jaw) or a dental (tooth) issue. Dentists don't routinely recommend the clear aligner option and make the presumption that some patients do not want to pay which is a mistake. If a patient in need of a stent for a blocked artery is not made aware of an anti-coagulant stent option, it is depriving that patient of a choice. In this case the choice is between a digital orthodontic appliance and an analogue appliance.

'Take a look back in history. Our parents had a metal ring around every single tooth. Our children are offered clear aligners. In 20 to 30 years, our grandchildren won't know what brackets are because they will be something of the past. I wrote a clinical orthodontic textbook in May 2018 called Clear Aligner Technique published by Quintessence which highlights how clear aligner technology has gained significant momentum in recent years and how I believe aligners are the future of orthodontics. My English textbook sold out in 14 months and was translated into seven languages within just a year. The translation in Russian, the eighth language, is coming soon. This is an indicator of the amount of interest in clear aligners amongst the dental profession. The textbook, 'the bible of aligners', starts off describing differences in ways aligners and brackets move teeth; then moves on with the software; and addresses all types of malocclusions and gives a protocol to follow.
'Clear aligners are a disruptive technology that challenges doctors about how to move teeth. We can control tooth movement using the software, just like your GPS is helping you to get from A to B, indicates traffic jams, and lets you know at what time you will arrive. The question is: would you drive without a GPS?'

For more information visit www.invisalign. co.uk.

\section{Biography:}

Dr Sandra Tai completed her graduate training in orthodontics at the University of Minnesota in Minneapolis where she received a Certificate in Orthodontics and a Master of Science degree. Currently, as a certified specialist in orthodontics and an Invisalign Top 1\% Provider who has treated over 2,000 cases with the Invisalign system, Dr Tai is a Clinical Assistant Professor in orthodontics at the University of British Columbia teaching both graduate and undergraduate orthodontic programs and she's the Coordinator of the Invisalign University Program. She's taught Invisalign at various universities worldwide, has lectured at universities across the globe, has been a presenter for different orthodontic associations and was the featured speaker at a number of Invisalign conferences.

In 2014, Dr. Tai was one of six members of the Clinical Review Committee for the Invisalign Gallery. She is part of the Align Tech Faculty in North America and is currently involved in several clinical research projects and FDA clinical trials with clear aligners. She still manages to find time to teach Invisalign Immersion Courses in her private practice.

Dr Tai is a Fellow of the College of Dental Surgeons of British Columbia and the Royal College of Dentists, Canada, was the founding member and past president of the Orthodontic Ties Study Club in Vancouver and maintains numerous professional affiliations. 